\title{
artigo
}

Gomes, A.F.; Yoshimura, B.K.; Souza, H.F.; Lima, N.R.; Paula, S.A.; Marques, M.V.;

A importância da consulta de enfermagem cardiológica na prevenção de doenças e promoção de saúde

\section{A importância da consulta de enfermagem cardiológica na prevenção de doenças e promoção de saúde}

The importance of cardiac nursing consultation in disease prevention and health promotion

La importancia de la consulta de enfermería cardíaca en la prevención de enfermedades y la promoción de la salud

\section{RESUMO}

A consulta de enfermagem pode ser realizada de maneira generalista ou com foco especialista. Objetivo: Identificar quais são os benefícios em se realizar a consulta de enfermagem com foco cardiológico para prevenção de doenças e promoção de saúde. Método: Revisão bibliográfica, tipo integrativa, nos idiomas português, inglês e espanhol, publicados nos últimos dez anos nas bases de dados indexadas (SCIELO, LILACS e MEDLINE) na BVS (Biblioteca Virtual da Saúde). Resultados: A consulta de enfermagem cardiológica permite: identificar precocemente os riscos que desenvolvem doenças cardiovasculares, melhorar a qualidade de vida dos pacientes por meio das orientações, otimizar recursos e sua melhor distribuição pelos níveis de saúde e valorizar o profissional. Conclusão: A consulta de enfermagem proporciona resultados positivos, empodera o trabalho do enfermeiro e permite planejar estratégias e medidas que atuam na prevenção e promoção de saúde.

DESCRITORES: Consulta de Enfermagem; Cardiologia; Cuidados de Enfermagem; Prevenção e Promoção.

\section{ABSTRACT}

The nursing consultation can be carried out in a general way or with a specialist focus. Objective: To identify the benefits of carrying out the nursing consultation with a cardiological focus for disease prevention and health promotion. Method: Bibliographic review, integrative type, in Portuguese, English and Spanish, published in the last ten years in the indexed databases (SCIELO, LILACS and MEDLINE) in the VHL (Virtual Health Library). Results: The cardiology nursing consultation allows early identification of risks that develop cardiovascular diseases, improve the quality of life of patients through guidelines, optimize resources and their better distribution by health levels and value the professional. Conclusion: The nursing consultation provides positive results, empowers the nurse's work, and allows planning strategies and measures that work in the prevention and promotion of health.

DESCRIPTORS: Nursing Consultation; Cardiology; Nursing care; Prevention and Promotion.

\section{RESUMEN}

La consulta de enfermería se puede realizar de forma general o con enfoque especializado. Objetivo: Identificar los beneficios de realizar una consulta de enfermería con enfoque cardiológico para la prevención de enfermedades y promoción de la salud. Método: Revisión bibliográfica, tipo integrativa, en portugués, inglés y español, publicada en los últimos diez años en las bases de datos indexadas (SCIELO, LILACS y MEDLINE) en la BVS (Biblioteca Virtual en Salud). Resultados: La consulta de enfermería cardíaca permite: identificar precozmente los riesgos que desarrollan las enfermedades cardiovasculares, mejorar la calidad de vida de los pacientes a través de guías, optimizar los recursos y su mejor distribución por niveles de salud y valorar al profesional. Conclusión: La consulta de enfermería arroja resultados positivos, empodera el trabajo de la enfermera y permite planificar estrategias y medidas que funcionen en la prevención y promoción de la salud.

DESCRIPTORES: Consulta de Enfermería; Cardiología; Cuidado de enfermera; Prevención y Promoción.

RECEBIDO EM: 13/01/2021 APROVADO EM: 25/01/2021

\section{Allan Francisco Gomes}

Enfermeiro graduado na Universidade Cidade de São Paulo com término em 2020.

ORCID: 0000-0002-5707-9026

\section{Beatriz Kaory Yoshimura}

Enfermeira graduada na Universidade Cidade de São Paulo com término em 2020.

ORCID: 0000-0003-3607-5533 


\section{Henrique Ferreira de Souza}

Enfermeiro graduado na Universidade Cidade de São Paulo com término em 2020. ORCID: 0000-0002-6125-0922

\section{Natalia Ribeiro de Lima}

Enfermeira graduada na Universidade Cidade de São Paulo com término em 2020. ORCID: 0000-0002-4948-9762

\section{Samuel Almeida de Paula}

Enfermeiro graduado na Universidade Cidade de São Paulo com término em 2020. ORCID: 0000-0002-1143-9532

\section{Marceli Vituri Marques}

Mestre em educação pela Universidade Cidade de São Paulo (UNICID- SP), Especialista em docência, administração hospitalar e ferramentas virtuais, graduada em Enfermagem pelo Centro Universitário São Camilo e docente a graduação na UNICID e pós graduação em cursos no PERU.

ORCID: 0000-0002-4378-287X

\section{INTRODUÇÃO}

0 país está em uma transição demográfica que nos leva a observar o perfil epidemiológico de algumas doenças em relação a idade, e conforme pesquisa realizada no DATASUS em março de 2020 em comparativo com alguns artigos publicados, o número de internações e mortes na população brasileira por doenças cardiovasculares nos idosos, vem crescendo ao longo dos anos. ${ }^{1,2,3}$

Dentre as principais doenças cardiovasculares estão: hipertensão, infarto agudo do miocárdio, angina de peito, doenças nas válvulas cardíacas, doenças cardíacas congénitas, endocardite, arritmias cardíacas, miocardite e tumores no coração.

A realização da consulta de enfermagem tem se tornado uma estratégia eficaz para a detecção precoce de riscos e acompanhamento de medidas instituídas. Conforme a lei 7498/86, a consulta de enfermagem, é uma atividade privativa do enfermeiro, que seguindo as etapas do processo de enfermagem é possível realizar a Sistematização da Assistência de Enfermagem (SAE). Consistem em identificar os problemas de saúde-doença, executar e avaliar os cuidados que contribuirão para a promoção, proteção, recuperação e reabilitação da saúde. ${ }^{4}$

A consulta de enfermagem viabiliza o trabalho do enfermeiro durante o atendimento, e pode ser realizada de maneira generalista ou com foco especialista, e proporciona orientação de medidas favoráveis que visam a abordagem apropriada as peculiaridades dos pacientes nas diferentes faixas etárias. ${ }^{5}$

Para a sua realização é necessário um local com materiais e equipamentos adequados e um ambiente confortável, privativo, seguro onde o profissional possa exercer seu ofício e o paciente consiga se sentir à vontade.

$\mathrm{O}$ relacionamento interpessoal permite criar um elo de confiança por meios de humanização, acolhimento, respeito, atenção e empatia, que auxiliam no levantamento de dados e na compreensão do paciente acerca do atendimento no processo saúde-doença.

De acordo com a Resolução COFEN $358 / 2009$, o processo de Enfermagem é um instrumento metodológico que orienta e documenta o cuidado de enfermagem. É constituído de cinco etapas as quais são interligadas: coleta de dados de enfermagem ou histórico de enfermagem, diagnóstico de enfermagem, planejamento de enfermagem, implementação e avaliação de enfermagem. ${ }^{7}$

$\mathrm{Na}$ coleta de dados, por meio da anamnese e exame físico, são identificados os denominados focos de atenção, que são as condições que o cliente/paciente apresenta: sinais vitais, hábitos, sinais e sintomas, histórico familiar e de patologias anteriores, queixas, medicações em uso, tratamentos anteriores entre outras.

Nesta fase é importante identificar os principais fatores de risco (predisposição genética, uso de álcool e cigarros, stress, sedentarismo, obesidade, dieta rica em sal, fatores socioeconômicos, etnia, idade, sexo, entre outros).

Faz parte do exame físico, a interpretação de dados dos sinais vitais: pressão arterial (valores e classificação conforme Sociedade Brasileira de Cardiologia), frequência cardíaca (ritmo), frequência respiratória (cansaço aos esforços) e presença de dor (escalas de comportamento e relato, dores aos esforços). A glicemia capilar também é realizada, pois a Diabetes Mellitus (DM) poderá potencializar as complicações cardiovasculares.

No exame físico, todas as propedêuticas devem ser utilizadas, com foco em: ausculta cardíaca, torácica e de carótidas, inspeção de face e jugulares, palpação de pulsos e edemas e avaliação do abdômen e função renal. Nos pacientes diabéticos é importante realizar a avaliação dos pés com o teste de monofilamento de Semmes- Weinsteins 10g, além da interpretação de dados de exames laboratoriais e de imagem, valores da classificação do índice de massa corporal, circunferência abdominal, risco cardiovascular e a Classificação de Framinghan.

São estas informações que proporcionarão os dados necessários para o planejamento da assistência de enfermagem. É importante a participação e envolvimento do usuário enquanto sujeito ativo de seu tratamento, para que haja uma conscientização do mesmo sobre sua condição de saúde atual, não o fazendo refém da prescrição profissional, e desenvolvendo autonomia para o processo de educação em saúde. 


\section{artigo}

É na etapa da avaliação de enfermagem que se verifica se as intervenções e ações de enfermagem realizadas alcançaram o resultado esperado, sendo as vezes necessário, mudanças ou adaptações aos cuidados8. Todo registro de paciente é organizado em forma de prontuários (manuais ou eletrônicos). ${ }^{9}$

Além da realização do plano de cuidados o Enfermeiro poderá orientar este paciente a participar de Programas dos SUS (Sistema único de Saúde) voltados para a prevenção de doenças cardiovasculares. São eles: Hiperdia, sistema que permite o cadastramento e o acompanhamento dos portadores de Hipertensão Arterial (HAS) e DM e ESF (Estratégia da Saúde da Família) que visam ações comunitárias os quais são: sistema de vigilância de fatores de risco e proteção para doenças crônicas não transmissíveis e pesquisas nacional de doenças crônicas, estilo de vida, consumo alimentar, uso de álcool, atividade física, tabagismo, acesso e utilização de serviço de saúde, utilização e promoção do uso racional de medicamentos e sistema de vigilância alimentar e nutricional.

As doenças cardiovasculares podem ser atendidas nos diferentes níveis de atenção. Caberá ao enfermeiro identificar, por meio da consulta de enfermagem, qual é a Instituição de Saúde que o paciente deverá realizar o acompanhamento.

Os principais cuidados em pacientes cardiológicos são voltados para a prevenção e manutenção da saúde, que de certa forma, visam conscientizar e mostrar que o próprio paciente é também responsável pelo seu cuidado e contam com estratégias que começam desde o diagnóstico e contribuem para a qualidade de vida de cada paciente abordado.

Sendo assim, o objetivo do trabalho é identificar nas referências científicas publicadas, quais são os benefícios em se realizar a consulta de enfermagem com foco cardiológico para prevenção de doenças e promoção de saúde.

\section{METODOLOGIA}

O trabalho consiste em uma revisão bibliográfica, do tipo integrativa, realizado por meio de pesquisa na BVS (Biblioteca Virtual da Saúde), utilizando as bases científicas Scientific Electronic Library Online (SciELO); Literatura Latino-Americana e do Caribe em Ciências da Saúde (Lilacs); e Medical Literature Analysis and Retrieval System Online (Medline).

A revisão bibliográfica é processo no qual é realizado a busca, análise e descrição de um determinado tipo de conhecimento em busca de respostas a inquietação específica por meio de base de dados cientificas composta por livros, artigos de revistas, artigos de jornais, registros históricos, teses e dissertações e outros tipos. ${ }^{12}$

A busca das referências se deu na data de 27/03/2020 até 30/04/2020. Foram encontrados um total de 20 artigos e 1 teses/dissertações/outros que após a análise crítica, foram utilizados 9 (nove) artigos e
01 tese de doutorado para a interpretação e a síntese dos dados coletados.

Os descritores foram definidos pela biblioteca virtual em saúde DeCS (Descritores em Ciência da Saúde): Consulta de Enfermagem; Cardiologia; Cuidados de Enfermagem; Prevenção e Promoção. Para a combinação desses DeCs, utilizou-se os operadores booleanos OR e AND. Foram incluídos estudos, publicados nos últimos cinco anos, disposição de textos completos nos idiomas português, inglês e espanhol, com o Brasil como país de origem.

Os estudos foram sintetizados de forma que evidenciassem os benefícios da consulta de enfermagem cardiológica para a prevenção e promoção de saúde.

\section{RESULTADOS}

Os dados obtidos foram agrupados $\mathrm{e}$ apresentados em forma de quadro, de modo a possibilitar uma melhor visualização dos estudos inseridos na revisão integrativa e bibliografica. Foram inseridas as seguintes informações: autores, ano de publicação, título, metodologia e ideia principal do texto. Os estudos do Quadro 1, estão na ordem em que aparecem nas referências.

\section{DISCUSSÃO}

De acordo com o artigo 1 do quadro de resultado, as doenças cardiológicas são um grande problema na sociedade atual, gerando gastos em todos os níveis de atenção à saúde: primário, secundário e terciário ${ }^{1,3}$ e que que os

Quadro 1. Caracterização dos estudos realizados no Brasil nos últimos dez anos, segundo autoria, ano de publicação, título, método utilizado e ideia principal dos estudos

\begin{tabular}{|c|c|c|c|}
\hline $\begin{array}{l}\text { AUTORIA E ANO DE } \\
\text { PUBLICAÇÃO }\end{array}$ & TÍTULO & METODOLOGIA & OBJETIVO \\
\hline $\begin{array}{l}\text { 1. Kernkamp CL, Costa } \\
\text { CKF, Massuda EM, } \\
\text { Silva ES, Yamaguchi UM, } \\
\text { Bernuci MP - }\end{array}$ & \multirow{2}{*}{$\begin{array}{l}\text { Perfil de morbidade e } \\
\text { gastos hospitalares } \\
\text { com idosos no Paraná, } \\
\text { Brasil, entre } 2008 \text { e } 2012 .\end{array}$} & \multirow{2}{*}{$\begin{array}{l}\text { As metodologias aná- } \\
\text { lise de componentes } \\
\text { principais (ACP) e análi- } \\
\text { se de agrupamentos }\end{array}$} & \multirow{2}{*}{$\begin{array}{l}\text { Analisar o perfil das morbidades e gastos hospitala- } \\
\text { res com idosos em relação às condições socioeconô- } \\
\text { micas e demográficas do Paraná, Brasil, no período } \\
\text { de } 2008 \text { a 2012, aplicando-se a análise fatorial em } \\
\text { componentes principais e de agrupamentos. }\end{array}$} \\
\hline $\begin{array}{l}\text { Ano de publicação de } \\
2016 .\end{array}$ & & & \\
\hline $\begin{array}{l}\text { 2.Gama GGG, Mussull } \\
\text { FC, Guimarães AC. }\end{array}$ & \multirow{2}{*}{$\begin{array}{l}\text { Revisando os fatores de } \\
\text { risco cardiovascular. }\end{array}$} & \multirow[t]{2}{*}{ Revisão de literatura } & \multirow{2}{*}{$\begin{array}{l}\text { Revisar a literatura para oferecer subsídios ao cuida- } \\
\text { do com a saúde. }\end{array}$} \\
\hline Ano de publicação 2010. & & & \\
\hline
\end{tabular}




\begin{tabular}{|c|c|c|c|}
\hline $\begin{array}{l}\text { 3.Massa KHC, Duarte } \\
\text { YAO, Chiavegatto Filho } \\
\text { AP. }\end{array}$ & \multirow{2}{*}{$\begin{array}{l}\text { Análise da prevalência de } \\
\text { doenças cardiovasculares } \\
\text { e fatores associados em } \\
\text { idosos, 2000- } 2010 .\end{array}$} & \multirow{2}{*}{$\begin{array}{l}\text { Pes } q \text { quisa de múltiplas } \\
\text { coortes com coleta de } \\
\text { dados de amostras } \\
\text { representativas }\end{array}$} & \multirow{2}{*}{$\begin{array}{l}\text { Analisar a mudança na prevalência de doença cardio- } \\
\text { vascular (DCV) entre } 2000 \text { e } 2010 \text { e sua associação } \\
\text { com os fatores de risco em idosos. }\end{array}$} \\
\hline $\begin{array}{l}\text { Ano de publicação de } \\
2019 .\end{array}$ & & & \\
\hline $\begin{array}{l}\text { 4,Castro Júnior AR, Abreu } \\
\text { LDP, Lima LL, Araújo AF, } \\
\text { Torres RAM, Silva MRF. } \\
\end{array}$ & \multirow[t]{2}{*}{$\begin{array}{l}\text { Consulta de Enfermagem } \\
\text { no cuidado ambulatorial às } \\
\text { juventudes. }\end{array}$} & \multirow[t]{2}{*}{$\begin{array}{l}\text { Estudo bibliográfico, } \\
\text { tipo revisão integrativa. }\end{array}$} & \multirow[t]{2}{*}{$\begin{array}{c}\text { Identificar as evidências cientificas sobre a consulta } \\
\text { de Enfermagem no cuidado ambulatorial às juven- } \\
\text { tudes }\end{array}$} \\
\hline Ano de publicação 2019. & & & \\
\hline $\begin{array}{l}\text { 5. Sobral MGV, Pessoa } \\
\text { VLMP, Florêncio RS et. }\end{array}$ & \multirow{2}{*}{$\begin{array}{c}\text { Elementos essenciais da } \\
\text { consulta de Enfermagem à } \\
\text { criança e ao adolescente }\end{array}$} & \multirow{2}{*}{$\begin{array}{c}\text { Estudo bibliográfico, } \\
\text { descritivo, tipo revisão } \\
\text { integrativa, }\end{array}$} & \multirow{2}{*}{$\begin{array}{l}\text { Identificar os elementos essenciais da consulta de } \\
\text { enfermagem na atenção à criança e ao adolescente. }\end{array}$} \\
\hline Ano de publicação 2018. & & & \\
\hline $\begin{array}{l}\text { 6. Santos LB dos, Lima } \\
\text { WL de, Souza JMO de et. }\end{array}$ & \multirow{2}{*}{$\begin{array}{l}\text { Risco cardiovascular em } \\
\text { usuários hipertensos da } \\
\text { atenção primária a saúde. }\end{array}$} & \multirow{2}{*}{$\begin{array}{l}\text { Estudo quantitativo e } \\
\text { transversal realizado } \\
\text { com } 115 \text { usuários } \\
\text { hipertensos. }\end{array}$} & \multirow{2}{*}{$\begin{array}{l}\text { Identificar os fatores associados ao risco de doenças } \\
\text { cardiovasculares em usuários da atenção primária à } \\
\text { saúde (APS) hipertensos. }\end{array}$} \\
\hline Ano de publicação 2018. & & & \\
\hline $\begin{array}{l}\text { 7. Asssunção CS, Rizzo } \\
\text { ER, Santos ME et. }\end{array}$ & \multirow{2}{*}{$\begin{array}{l}\text { O Enfermeiro no pré-natal } \\
\text { Expectativas de Gestante. }\end{array}$} & \multirow{2}{*}{$\begin{array}{c}\text { Pesquisa de campo } \\
\text { de cunho qualitativo, } \\
\text { exploratório descritivo. }\end{array}$} & \multirow{2}{*}{$\begin{array}{c}\text { Buscar evidências, aprofundar o conhecimento } \\
\text { sobre a temática e descrever sobre a expectativa } \\
\text { da gestante quando o enfermeiro é inserido em seu } \\
\text { pré-natal. }\end{array}$} \\
\hline Ano de publicação 2018. & & & \\
\hline $\begin{array}{l}\text { 8. Girondi JBR, Fernandez } \\
\text { DLR, Silvestrin ANS et. }\end{array}$ & \multirow{2}{*}{$\begin{array}{l}\text { Implantação da consulta } \\
\text { de enfermagem num } \\
\text { centro endoscópico. }\end{array}$} & \multirow{2}{*}{$\begin{array}{c}\text { Trata se de estudo } \\
\text { descritivo qualitativo, } \\
\text { do tipo relato de caso } \\
\text { realizado em um centro } \\
\text { endoscópico. }\end{array}$} & \multirow{2}{*}{$\begin{array}{c}\text { Relatar a experiência de implantação da Consulta de } \\
\text { Enfermagem no serviço de endoscopia do Hospi- } \\
\text { tal Universitário da Universidade Federal de Santa } \\
\text { Catarina. }\end{array}$} \\
\hline Ano de publicação 2019. & & & \\
\hline $\begin{array}{l}\text { 9. Santesso ACOA, Frie- } \\
\text { drich DBC. }\end{array}$ & \multirow{2}{*}{$\begin{array}{l}\text { Desinformação do usuário } \\
\text { e oportunidade para a } \\
\text { enfermagem. }\end{array}$} & \multirow{2}{*}{$\begin{array}{l}\text { Estudo qualitativo, do } \\
\text { tipo descritivo e com- } \\
\text { parativo. }\end{array}$} & \multirow{2}{*}{$\begin{array}{c}\text { Analisar as repercussões imediatas da ação edu- } \\
\text { cativa do enfermeiro realizada na sala de espera da } \\
\text { hemodinâmica, voltada aos pacientes e acompa- } \\
\text { nhantes, antes de um procedimento de intervenção } \\
\text { cardiovascular. }\end{array}$} \\
\hline Ano de publicação 2016. & & & \\
\hline $\begin{array}{l}\text { 10. Moreira DS. } \\
\text { Tese de doutorado }\end{array}$ & \multirow[b]{2}{*}{$\begin{array}{l}\text { Consulta de Enfermagem } \\
\text { a pessoa com doença car- } \\
\text { diovascular: Valorizando as } \\
\text { habilidades empáticas. }\end{array}$} & \multirow{2}{*}{\begin{tabular}{|c|} 
Pesquisa com abor- \\
dagem qualitativa \\
descritiva, devido a \\
indispensabilidade \\
de se aprofundar nas \\
necessidades e desejos \\
na perspectiva da \\
pessoa atendida.
\end{tabular}} & \multirow[b]{2}{*}{$\begin{array}{l}\text { Valorização das habilidades empáticas na consulta } \\
\text { de enfermagem a pessoa que convive com doença } \\
\text { cardiovascular. }\end{array}$} \\
\hline Ano de publicação 2018. & & & \\
\hline
\end{tabular}

distúrbios cardiológicos são problemas que, na maioria das vezes, são previsíveis. ${ }^{2,3}$

$\mathrm{O}$ artigo 2 da tabela de resultados, (Revisando os fatores de risco cardiovascular, 2010) complementa que nove são os fatores responsáveis por mais de $90 \%$ das doenças cardiovasculares, sendo que, destes seis atuam de forma prejudicial (dislipidemia, hipertensão arterial, diabetes sobrepeso/ obesidade, tabagismo e estresse psicológico) e três de forma protetora (exercício físico, consumo diário de vegetais, frutas e bebidas alcoólicas em pequena dose). ${ }^{2}$
Sendo assim, a enfermagem tem uma grande atuação no processo saúde-doença, pois poderá atuar nos dois extremos: antes ou após a instalação de um problema de saúde. ${ }^{4,5}$ É na consulta de enfermagem, com a realização das etapas de coleta de dados (anamnese e exame físico), que é possível identificar fatores de risco e assim planejar e implementar estratégias de cuidados para prevenir agravos e promover saúde. ${ }^{4,5}$

Em conformidade aos estudos interpretados, a consulta de enfermagem cardiológica permite: identificar precocemente os riscos que desenvolvem doenças cardiovasculares, melhorar a qualidade de vida dos pacientes por meio das orientações, otimizar recursos e sua melhor distribuição pelos níveis de saúde e valorizar o profissional. ${ }^{4,67,7,89}$

O estudo 9 (Desinformação do usuário e oportunidade para a enfermagem, 2016) destaca que o enfermeiro tem responsabilidade na elaboração de estratégias que ampliem sua visibilidade e mostre aos seus pacientes que eles mesmos devem compreender a sua importância como participante ativo no seu próprio cuidado, pois pre- 


\section{artigo}

Gomes, A.F.; Yoshimura, B.K.; Souza, H.F.; Lima, N.R.; Paula, S.A.; Marques, M.V.i

A importância da consulta de enfermagem cardiológica na prevenção de doenças e promoção de saúde

venção e promoção podem ser sinônimo de qualidade de vida e melhora de uma sociedade, mas sobretudo a continuação de um trabalho que ao longo dos anos trarão bons resultados?.

Deste modo o enfermeiro deve estar capacitado e ciente do quão importante será cada passo dado dentro da avaliação cardiológica, além de compreender e utilizar o diagnóstico de enfermagem de maneira correta, pois é um papel importante dentro do processo de enfermagem afim de obter ideias e coletar dados com a responsabilidade clínica.

Assim é realizado um planejamento e desenvolvimento nas funções do enfermeiro para proporcionar e otimizar uma qualidade clínica, concluindo com a evolução de enfermagem. Todas essas etapas são importantes para avaliar o quadro clínico do paciente, sendo objetivo e coerente para produzir uma ordem de acontecimentos.
A partir do que é coletado e discutido sobre cada paciente é possível prevenir doenças e alcançar uma prevenção próxima relacionado ao histórico familiar.

\section{CONCLUSÃO}

A consulta de enfermagem viabiliza o trabalho do enfermeiro durante o atendimento, ela pode ser realizada de maneira generalista ou com foco especialista, e proporciona orientação de medidas favoráveis que visam a abordagem apropriada as peculiaridades dos pacientes nas diferentes faixas etárias.

$\mathrm{O}$ enfermeiro pode gerar um roteiro de avaliação para anamnese e exame físico e após a partir dos dados coletados, descrever os principais diagnósticos de enfermagem (com referência apropriada para cada serviço), que facilitarão no planejamento, imple- mentação e avaliação dos cuidados de enfermagem prescritos.

Hoje segundo a Política Nacional de Humanização é valido utilizar de uma proposta de clínica ampliada com multiprofissionais e principalmente com a participação de pacientes e familiares para que eles um olhar ampliado sobre as facetas que permeiam adesão).

É possível evidenciar com todos os estudos realizados a importância de se discutir cada vez mais o tema em questão. Ao longo dos anos de estudos acadêmicos, tornou-se perceptível que a enfermagem além de vinculada a prestação de cuidados, pode e deve fortalecer a profissão com a produção de pesquisas científicas.

A consulta de enfermagem proporciona resultados positivos, empodera o trabalho do enfermeiro e permite planejar estratégias e medidas que atuam na prevenção e promoção de saúde.

\section{REFERÊNCIAS}

1. Kernkamp CL, Costa CKF, Massuda EM, Silva ES, Yamaguchi UM, Bernuci MP. Perfil de morbidade e gastos hospitalares com idosos no Paraná, Brasil, entre 2008 e 2012, Cad. Saúde pública vol. 32 n 7 Rio de janeiro.2016; Acesso em 31/03/2020; Disponivel em: https://www.scielo.br/scielo.php?script=sci_arttext\&nrm=iso\&lng=pt\&tlng=pt\&pid=S0102-311X2016000705005.

2. Gama GGG, Mussull FC, Guimarães AC. Revisando os fatores de risco cardiovascular, Rev. enf. UERJ,2010; online acesso em 27/02/2020. Disponivel em: https://www.facenf.uerj.brv18n4/ v18n4a25.

3. Massa KHC, Duarte YAO, Chiavegatto Filho AP, Análise da prevalência de doenças cardiovasculares e fatores associados em idosos 2000-2010, Rev. Ciênc. Saúde coletiva vol.24 no.1 Rio de Janeiro. 2019; online, acesso em 30/03/2020. Disponivel em: https://www.scielo.br/scielo.php?script=sci_arttext\&n$r m=i s o \& l n g=p t \& t$ lng=pt\&pid=S1413-81232019000100105.

4. Castro Júnior AR, Abreu LDP, Lima LL, Araújo AF, Torres RAM, Silva MRF, Consulta de enfermagem no cuidado ambulatorial a juventude, Rev. Enf. UFPE, online, Recife. 2019; acesso em 27/03/ 2020. Disponivel em:https://periodicos.ufpe.br/revistas/ Revistaenfermagem/article/view/239115/31854

5. Sobral MGV, Pessoa VLMP, Florêncio RS et, Elementos essenciais da consulta de enfermagem à criança e ao adolescente, Rev enferm UFPE online, Recife 2018, acesso 28/03/2020, disponivel em: https://pesquisa.bvsalud.org/portal/resource/pt/ biblio-1005311.

6. Santos LB dos, Lima WL de, Souza JMO de et, Risco cardiovascular em usuários hipertensos da atenção primária à saúde, Rev. enferm. UFPE online, Recife 2018, acesso 27/03/2020. Di- sponivel em: https://pesquisa.bvsalud.org/portal/resource/pt/ biblio-980604.

7. Moreira, Diego da Silva, Consulta de Enfermagem à pessoa com doença cardiovascular: valorizando as habilidades empáticas / Nursing consultation to the person with cardiovascular disease: valuing the empathic abilities, Rio de Janeiro; s.n; 2018, acesso 28/03/2020. Disponivel em: https://pesquisa.bvsalud. org/portal/resource/pt/biblio-1024187.

8. Asssunção CS, Rizzo ER, Santos ME et, O Enfermeiro no pré-natal: Expectativas de Gestantes, Ver Fund Care Online, 02/04/2019, acesso 28/03/2020; Disponivel em: http://dx.doi. org/10.9789/2175-5361.2019.v11i3.576-581.

9. Girondi JBR, Fernandez DLR, Silvestrin ANS et, Implantação da consulta de Enfermagem Num Centro Endoscópico, Cienc Cuid Saude, 18/10/2018, acesso 30/03/2020; Disponivel em: https:// pesquisa.bvsalud.org/portal/resource/pt/biblio-974858.

10. Santesso ACOA, Friedrich DBC, Desinformação do usuário e oportunidade para enfermagem, Rev enferm UFPE online, Recife 2017, acesso 27/03/2020. Disponivel em: https://pdfs.semanticscholar.org/5873/be218d42a3588509b1abba4eb04dde3dc1fd.pdf

11. Ministério da saúde, data sus, brasil 2020, acesso em 27/03/2020, disponivel em:https://datasus.saude.gov.br/mortalidade-1996-a-2017-pela-cid-10-2/.

12. Faculdade de ciências agronômicas UNESP, Campus de Botucatu-biblioteca prof. Paulo de carvalho Mattos, Botucatu 2015, acesso em 23/05/2020, disponivel em: https://www.fca.unesp. br/Home/Biblioteca/tipos-de-evisao-deliteratura.pdf 